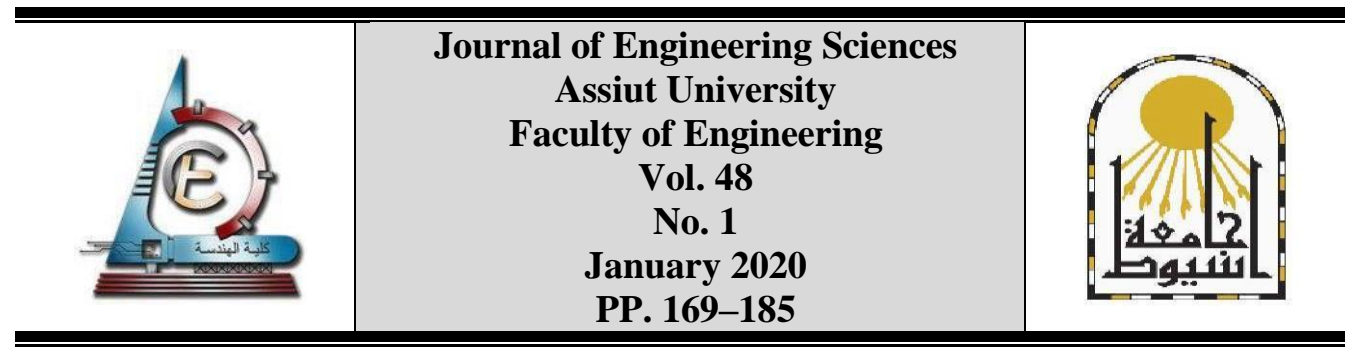

\title{
MATHEMATICS AS A DESIGN TOOL: TOWARD AN EFFECTIVE BUILDING'S AESTHETICAL PERFORMANCE
}

\author{
Aya M. Fawzy, Inas Abdel Sabour * \\ Architecture Department, Helwan University. \\ Received 2 July 2019; Accepted 27 August 2019
}

\begin{abstract}
Integrating mathematics in architectural design and construction helped in generating various design solutions. Thus, aesthetical performance had developed with the involving of advanced mathematics fields and computational software. The main intention of this paper is to study the importance of mathematics in aesthetical architecture performance. Consequently, the research methodology carries out an analysis of different case studies that offered a better perception about the degree of mathematics effectiveness on the aesthetical performance in architecture. The research will investigate to what extent mathematics forms in architecture had actually a great effect on aesthetical architecture performance.
\end{abstract}

Keywords: Mathematics, Geometry, Parametric, Algorithm, Topology, Aesthetic Performance.

\section{Introduction}

Mathematics has a significant effect on architecture by assisting designers in defining extraordinary shapes and designs. Thus, it became a central core in the designing process, from the initial stage of form finding, shaping and generating complex forms to the process of manufacturing, which opened new aesthetic horizons in the architecture field. Architects were mathematicians, and mathematicians were often architects, thus architecture and mathematics have many homogenous themes, by presenting many architects who discovered and used mathematical structures and patterns [1,2].

In architectural design, the performance concerns with three topics: Structural performance; that linked directly to the occupants' safety under the shelter of the building. Environmental performance; related to items that effected on the built environment's quality, both indoor and outdoor. Aesthetic and cultural performance; which was selected in this research, that affected by form, space's organization, material selection, color, and shape, that played a role in forming the aesthetic and cultural performances of a building [3,4].

\subsection{Research aims}

The study aimed to analyse and measure the relationship between mathematics and aesthetical architecture performance by;

* Corresponding author.

E mail address: inas.abdelsabour@m-eng.helwan.edu.eg 
- Clarifying mathematical aspects and identifying each one's application in the architecture field.

- Prove that the mathematics has an essential role in promoting building's aesthetical performance.

\subsection{Research hypothesis}

The applying of mathematics as a design tool, it can offer a wide range of novelties variable forms within architectural work and promote the designer's creativity. Besides, it can be utilized in different ways to promote building's aesthetical performance.

\subsection{Research methodology}

With the intention of the objectives, the methodology is divided into two parts; Firstly, a review of related literature of mathematics via declaring its types, branches and application in architecture, then stating the factors of aesthetic performance concepts and identify its evaluation levels in architecture. Secondly, by using a designed questionnaire, then analysis, discussion and evaluation via selecting case studies to investigate to what extent the mathematical fields could promote the aesthetical performance, as in Fig. 1.

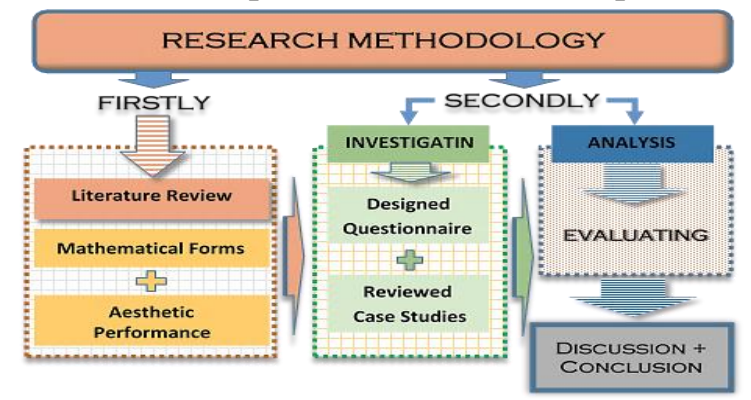

Fig. 1. Research Methodology [Author]

\section{Literature review}

A close connection between mathematics and architecture are limitless. Previously, many studies had explored the mathematical fields and its utilization in architecture, and the aesthetic performance evaluation subjects, and how we can apply the different mathematical types in achieving the optimal aesthetic performance.

\subsection{Historical review}

Such as, Ancient Egyptians and Indians architects who used the principles and percentages by using complex calculations to attain the building's dimensions and components, that imbedded the buildings with the space, by considering the sun and star's movements [5]. In addition, in Greek architecture, the Golden rectangle (1: 1.618) served as a rule for planning, that were based on considerations of aesthetics such as rhythm. While in $18^{\text {th }}$ and $19^{\text {th }}$, symmetry was used as a guiding principle, such as at the Taj Mahal that was based on the proportion of 1:2 that was reached only via mathematical calculations $[6,7]$. As well in the beginning of the $20^{\text {th }}$, the heightened use of rectilinear geometry appeared in modern architecture [7]. Then, in recent times, the fractal's concept was used to prove that such buildings have universal appeal and was visually attractive due to the ability of providing a sense of scale at different levels of viewing, as in Centre for Ideas [8] and Bogota International Convention Centre [9]. 


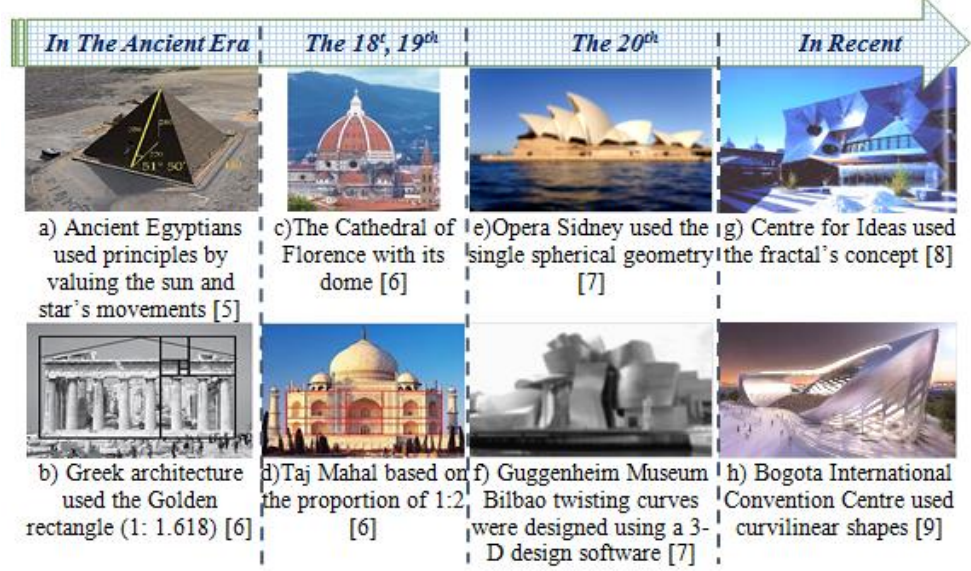

Fig. 2. Time Line of Using Of Mathematics in Architecture

\subsection{Mathematics in architecture.}

\subsubsection{Architecture design tool}

Architectural forms were created from the form generation process and conception, which was conditioned as an interdependent link between two factors; the aesthetic and technological ones, so it can be defined as a process that balances the intangible feature with the materializing aspect. These factors are dependent on the digital technology progress and on the evolution of mathematical concepts. The exploration of new mathematical methods is renovating the geometrical concepts in architecture has been strongly aided by the implementation of digital technology, as seen in Fig.3 [10].

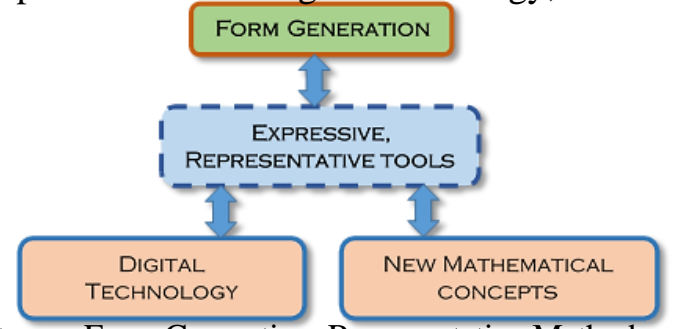

Fig. 3. Relationship between Form Generation, Representative Methods, and Influencing Factors [10]

\subsubsection{Mathematics as a design tool}

A new wave of interest in the relationship of mathematics to space making has been aesthetically driven, by defining the relation of numbers to architecture. Numbers gave us the dimensions and proportions of shapes, and add innovative meanings to buildings, by playing a vital role in architecture creation from ancient cultures, until the contemporary movements. Mathematics can be seen as a common science of structures by considering systems of elements and their relations or processes [11].

\subsubsection{Mathematical types}

The mathematics' importance in architecture was studied via many aspects, that helped in designing freeform architecture. On the other hand, some concerned with studying the harmonic relation between geometry and architectural design to keep the background of culture and understand the actual combination between geometrical thinking and architectural designing.

In consequence, from many previous studies in various applications, it could be classified into; Geometry, Parametric, Algorithm, and Topology. 


\section{Geometry}

Mathematics and geometry could be seen as a special study of structures by counting collective sets of architectural elements and their relations as well as operations. Thus, studying of geometry makes an important effort to design process by dealing with exploring of geometric figures, forms and shapes as elements and proportions, differences, angles positions and transformations as relations between them [12,13].

\section{- Euclidean Geometry}

It has many roots in measurements; therefore, it corresponds with the tactile space. It has just described the golden section as a continuous division. The objects could be classified in one, two or three dimensions, and had features that can be both planar and three dimensional, which is a mathematical expression that shows the space filling capacity of a pattern [10, 12, 13].

\section{- Non-Euclidean Geometry}

Currently, the using of Non-Euclidean geometry is an important way to developing the optimum structural forms and in searching for effective engineering solutions [14].

\section{- Discrete Differential Geometry}

It was a new and active mathematical field, where differential geometry (providing smooth surfaces) interacts with discrete geometry (concerned with polytopes, simplicial complexes, etc.), by using tools and ideas from all parts of mathematics. The study of meshes with offsets led to a new curvature theory for discrete surfaces based on parallel meshes, as; planar quad meshes, triangle mesh, conical mesh, and circular mesh as a basic surface representation $[15,16]$.

\section{- Fractal Geometry}

The use of fractal geometry as a design tool represents a completely new approach. It is a new branch of mathematics used to describe and analyse the varying shapes observed in natural structures the most common fractals can be constructed via perimeters of iterative patterns involving the creators of iterative functions on metric spaces, which considered a powerful mathematical tool that can be used to create fractals $[13,14,17,18]$.

\subsection{Parametric}

Parametric design is a process based on algorithmic thinking that allows the aspect of parameters and rules that define, clarify and encode the relationship between design aim and response. The concern of changing forces such as climate, setting, culture, and using has always formed part of the design process. It is powerful of owing the ability to capture a high degree of variation in a few numerical values, by involving an essential expertise of scripting of the mathematical algorithms whereby the interaction design is not possible $[1,13]$.

\subsection{Algorithm}

An algorithm is mathematics' term, which means a finite set of orders to attain a specific purpose in a clear way [19]. The inputs in the digital algorithms were the pattern and the number of repetitions [10]. The mathematical processing quality of the computeraided drafting and design software made algorithmic design an integral part of the architecture. The main advantage of using digital generators for optimal design results is the ability to model multi-variant solutions, and modifying easily by implementing numerical data iterations [20]. Besides, algorithms able to solve a wide range of 
Aya M. Fawzy, Inas Abdel Sabour, Mathematics as a design tool: toward an effective .........

computational problems, including sorting and searching, data structure operations, combinatorial or numerical problems and computational geometry [21].

There is a confusion that algorithmic design is synonymous with parametric design, but an algorithmic design does not have to be parametric at all while a parametric design is always an example of algorithmic design [22].

\subsection{Topology}

It explored the form as a process and not a finite condition, via adjustable mathematical functions or homeomorphism that proposed an interesting metaphoric formal concept "the fluidity of the form" [10].

Table. 1 shows the branches that have been used in architecture in details with clarifying the main differences between them and its application in architecture.

\section{Table 1.}

Types of Mathematics in architecture

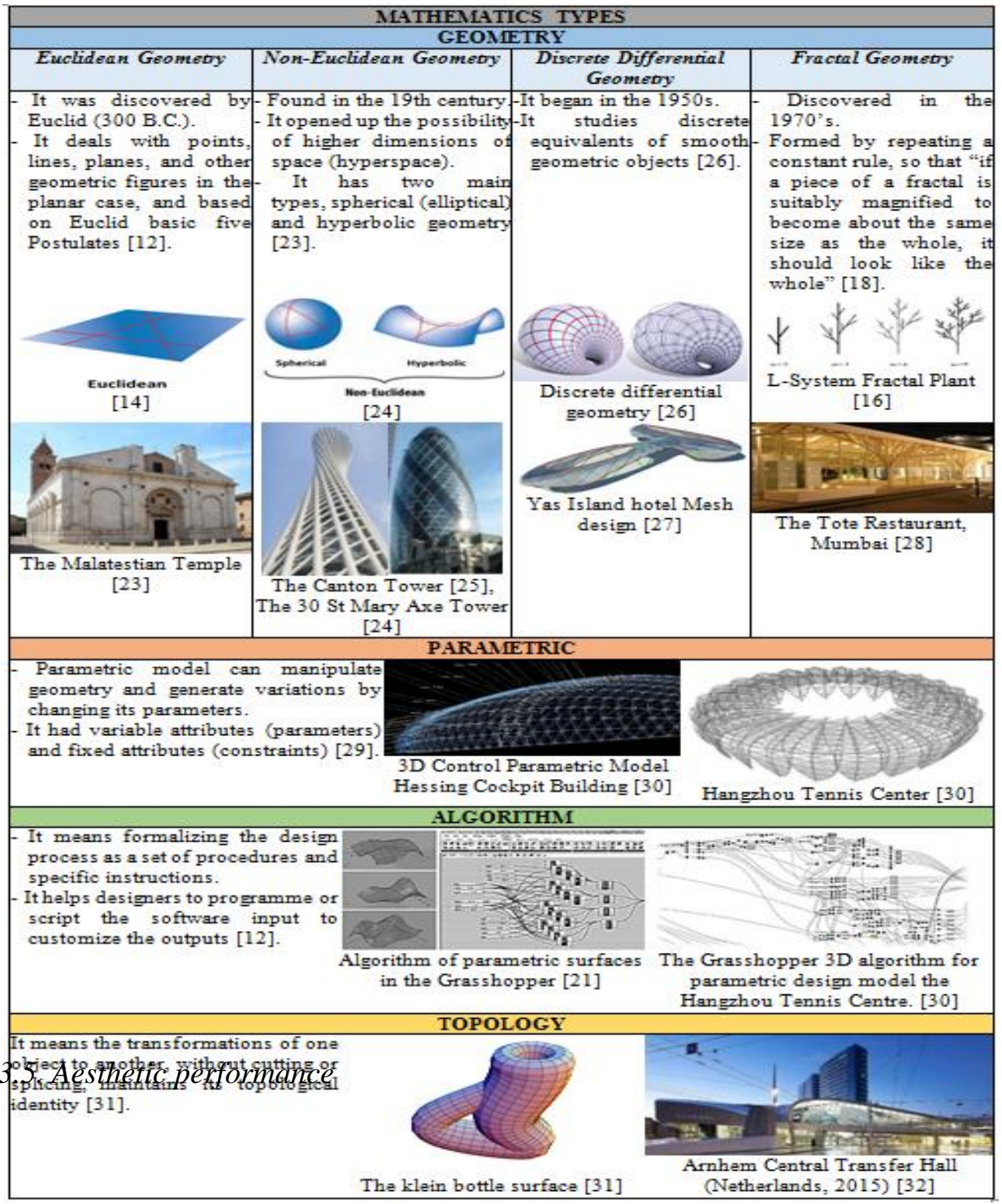


Aesthetic performance was as a main concern in the architectural design, and was considered a vital factor in the architectural design's value-system, it was determined via form, organization of space, material selection, colour, and shape. Quantifying Aesthetic performance is often difficult as it depends on many factors, and sometimes, it becomes a matter of personal preference or taste. Thus, researches try to accomplish the architectural aesthetic evaluation criteria and the weight of each element of quantification analysis, which was used to help in identifying the aesthetic factors.

In consequence, it could be classified into; The Environmental Performance, The Vision and Perception Performance, The Form and Beauty Performance and The Implication and Meaning Performance, as seen in [33,34].

The following are the concerns that can be used to determine aesthetic performance, and each one contains sub factors, which help in identifying its importance;

\subsubsection{The Environmental Performance.}

- The Integration of Architecture and Environment; it depends on the connection between architecture and environment such as; function, economy, technology, art, and culture. Also, the integration of the architecture's internal space by reflecting the natural environment.

- Architectural Modelling; by utilizing architectural modelling to unify environmentally the architectural shape and performance.

\subsubsection{The Vision and perception performance}

- Texture Performance; That focused on the usage of diverse materials that enrich a sense of vision and perception, by an understanding of the formation and response of its aesthetics.

- Light Performance; which influenced on displaying the shape and creating the architecture's atmosphere.

- Colour Performance; means the adoption of colour to the regional climate and the environment. Besides, the colour harmony with the city to demonstrate the nature of the architecture.

\subsubsection{The Form and beauty performance}

- Contrast and Slight Differ; Contrast emphasize on the multiplicity and change in sense of aesthetics. Whereas, slightly differ is micro-contrast, emphasizing on unification, and the combination of forms in an organic whole full of harmony and change.

- Balance and Stability; focused on the sense of psychological balance and stability that support form beauty and bring peace to the people.

- Diversity and Unification; diversity reflects features of the different architecture, while unification is the harmonious coordinator of all the architecture's components.

- Rhythm and Rhyme; that concerned with the phenomena of appearing regularly and repeating the changes systematically. Thus, the rhythm of aesthetic form divides into; continuous rhythm, the rhythm of the gradient, undulating rhythms, and staggered rhythms.

- Size and Scale; The harmonious ratio and relationship of all 3D's architectural components (length, width and height). There are types of scale impression which varied into; natural scale, exaggerated scale and gracious scale. 


\subsubsection{The Implication and meaning performance}

- City Landmark; that implies on individual sign of the city, which symbolizes its cultural features by verifying the history's development and the city's arts. It had three features; having familiar place to the users in everyday life, having special significance, and using a main position in the sense of vision.

- Mapping Function; The architecture with different function should demonstrate different emotional atmosphere.

- Psychological Implication; It represents people's psychological tendency such as; the link between the environment and behaviour, the environmental protection, and the utilization of the environment and space.

- Symbolic and Metaphorical; Symbolic states the cultural features of the national and regional via the architecture's space. While Metaphorical implies the context between the architecture, traditional culture, nature and history.

- City Context; It emphasizes on the history's development, culture, and environment in architecture, by integrating and adapting of the surrounding environmental characteristics and inheritance.

\section{Application method}

This part of the research aimed to determine to what extent mathematics shapes (Geometry, Parametric, Algorithmic and Topology) could attain the aesthetic performance factors, through an analytical study by the subsequent stages:

\subsection{The questionnaire strategy/development}

A standardized questionnaire was designed in a Google Form application, that was divided into four parts, each one indicates a type of mathematics forms by using visible figures of the six selected buildings for evaluating from the questionnaire takers' perspective. The questionnaire takers were selected according to their architectural knowledge degree; architects, lecturers, associate professors, and were asked to identify which factor could the building attain, according the building's description, 37 questionnaires were received from total 75 questionnaires. Then, the research was organizing the survey data, then accomplishing the statically analysis.

\subsection{Criteria of selecting case studies}

The case studies were selected consistent with the four mathematical types (Geometry, Parametric, Algorithmic and Topology). The variety of the building's function, size and the used materials, aids to study which of building forms will be more motivated by the scope of the research.

By benefitting from the above criteria, the research utilizing the analytical study to evaluate case studies and their success to express the building aesthetic performance via the proposed criteria. The results will display the degree of succeeding the mathematical types to attain the aesthetic performance, which help in offering a guideline paradigm to identify the proper shape type that can express the building's aesthetic performance. Table. 2 shows the selected 24 case studies that classified into four groups according to its shape. 
Table. 2.

The Selected Case Studies with Description

\section{GEOMETRY}

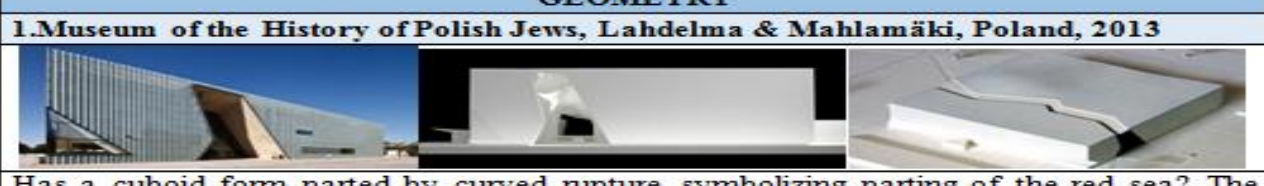

Has a cuboid form parted by curved rupture, symbolizing parting of the red sea? The Discrete differential geometry was used to design the quad's panels with double curved mesh that shaped the ruptured wall surface [35]

2.Yas Hotel, Asymptote Architecture, Abu Dhabi, UAE 2009

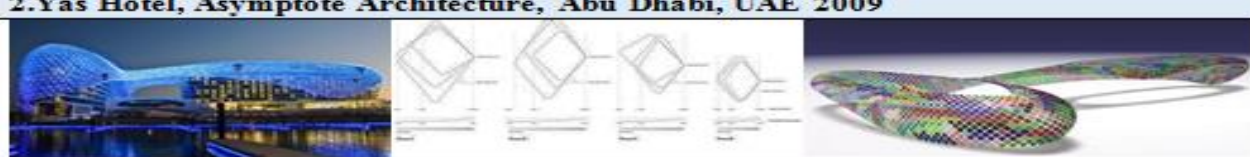

Had a Grid Shell with a curvilinear steel diamond shape. Discrete differential geometry was used to design "a torsion-free nodes" mesh that integrate the shell freeform shape and its structure beams [27]

3.The Canton Tower, Information Based Architecture, Guangdong, China, 2010

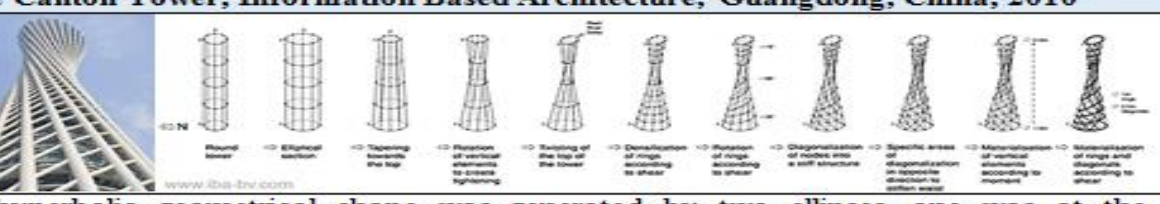

The hyperbolic geometrical shape was generated by two ellipses, one was at the foundation level, while the other raised to level $450 \mathrm{~m}$, creating the tower unique torsion, in $600 \mathrm{~m}$ tall, with 106 levels [25]

4.Melaku Centre Ethiopia, xvstudio, Ethiopia, 2009
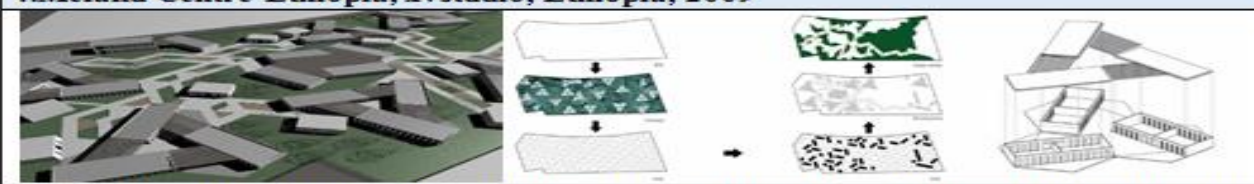

The project master-plan geometry is based on a fractal hexagonal grid that holds the different sizes of classrooms and services, a common theme in most of traditional African architecture is the use of fractal scale [36]

5.The Shell House, Kotaro Ide - ARTechnic architects, Japan, 2008

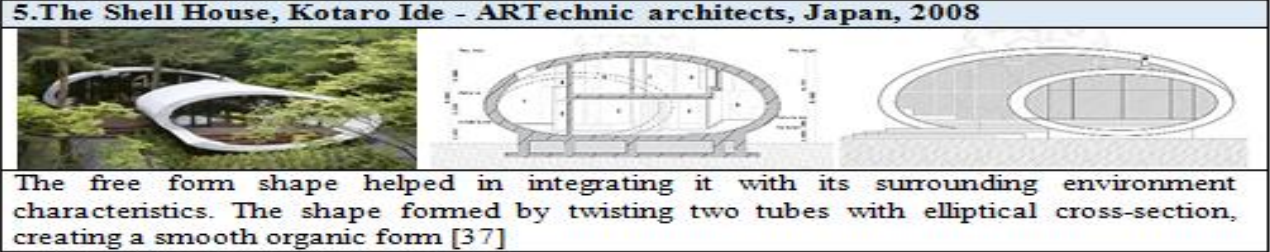

6.Grand Egyptian Museum, Heneghan Peng Architects, Cairo, 2018

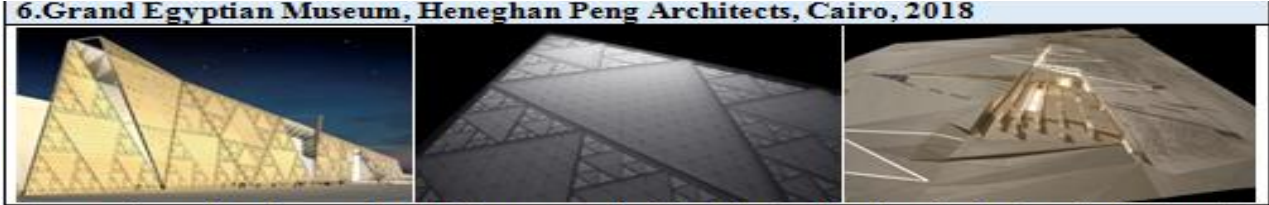

Its exterior walls a dopt a fractal element called the Sierpinski triangle that inspired from the great pyramid form [38]

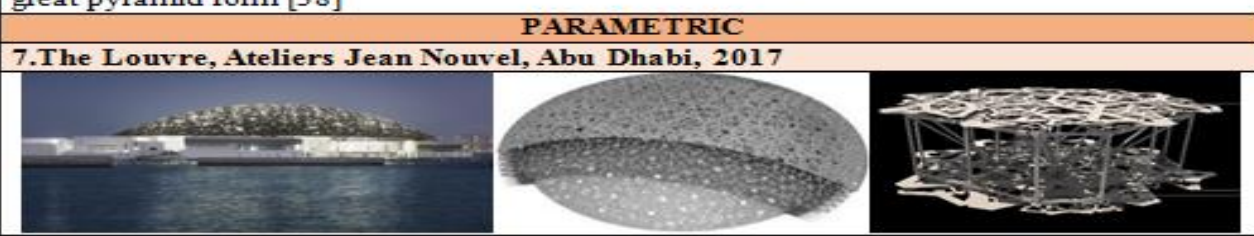

Represented the country history, culture and geography. The dome's geometric pattem takes up the Islamic tra dition of the perforated screen and fractal organization of pattering, creating a special micro-climate below and integrating with its surrounding natural environment [11] 
Table. 2. (Cont.)

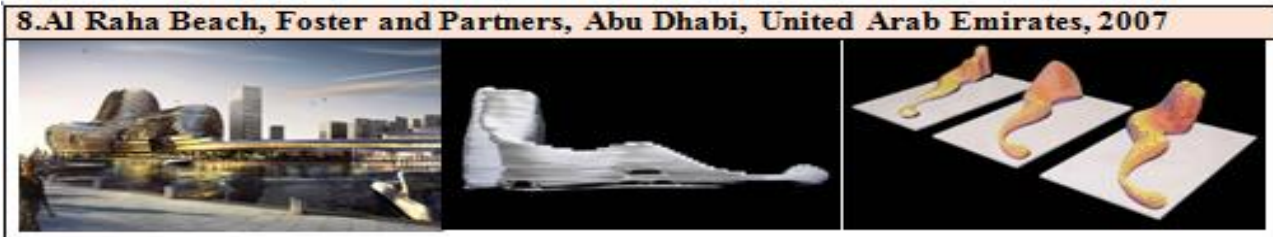

The design based on the interaction of sun and wind throughout the year into numerical data o help in forming the building shape, a louvre system minimizes solar gain, maximizes iews out, provides shade façade [39]

\section{Harbin Opera House, MAD Architects, China, 2015}

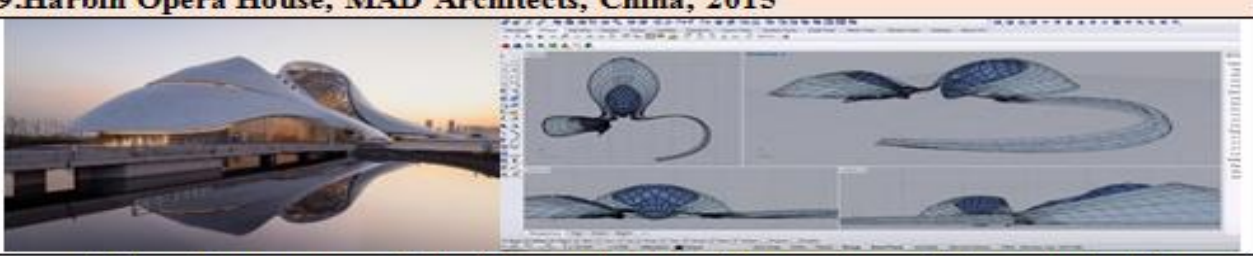

It was designed in response to the force and spirit of the northem city's natural wildemess and icy climate. Appearing as if sculpted by wind and water, the building seamlessly blends in with nature and the topography, a transfusion of local identity, art, and culture [40]

10.South Medical Research Institute, Woods Bagot, Australia, 2014
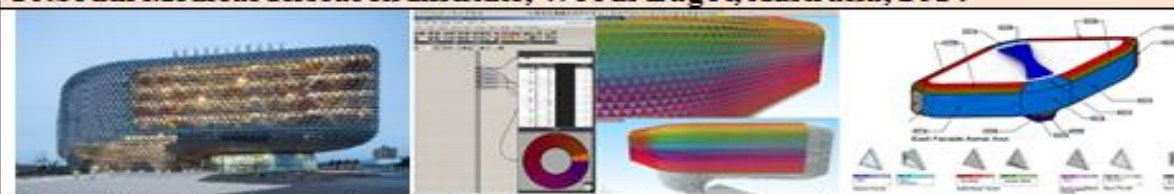

The building's unique triangulated diagrid facade responds to its environment like a living organism, the facade is designed to improve access to daylight, reduce heat and glare, and maintain vision for a healthy internal environment [41]

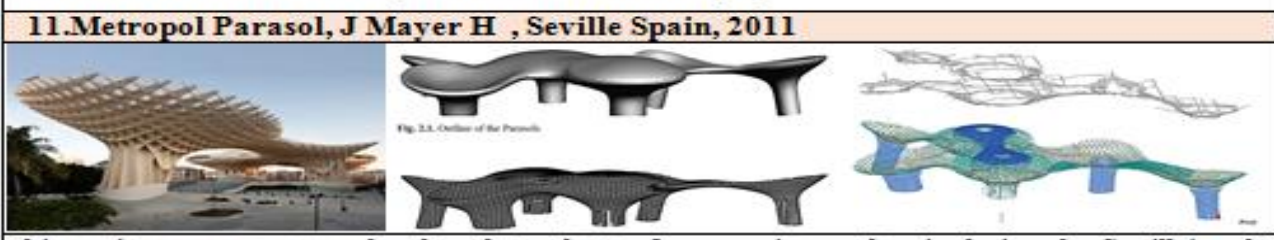

this project acts as a new landmark, a place of connecting and articulating the Seville's role as one of the world's most fascinating cultural destinations, it explores the potential of the Plaza de la Encamacion to become the new contemporary urban centre [42]

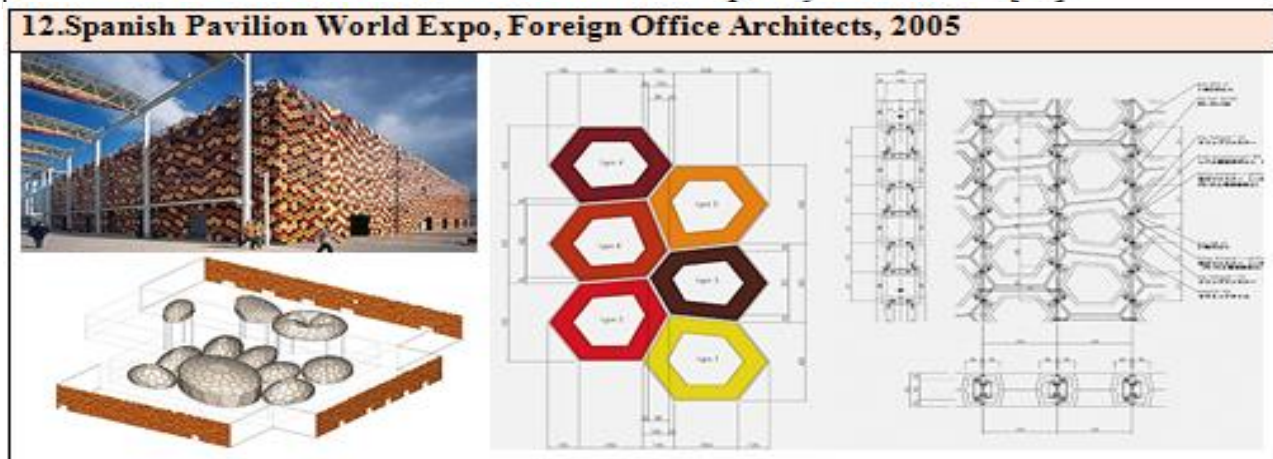

The pavilion focused on the Spanish cultural tradition. By lattice envelope that was its nonrepetitive pattem which used six hexagonal tile pieces (like many gothic and Islamic traceries), each of which were differently shaped and coded a different colour, maximizing the visual effects of the pavilion [43] 
Table. 2. (Cont.)

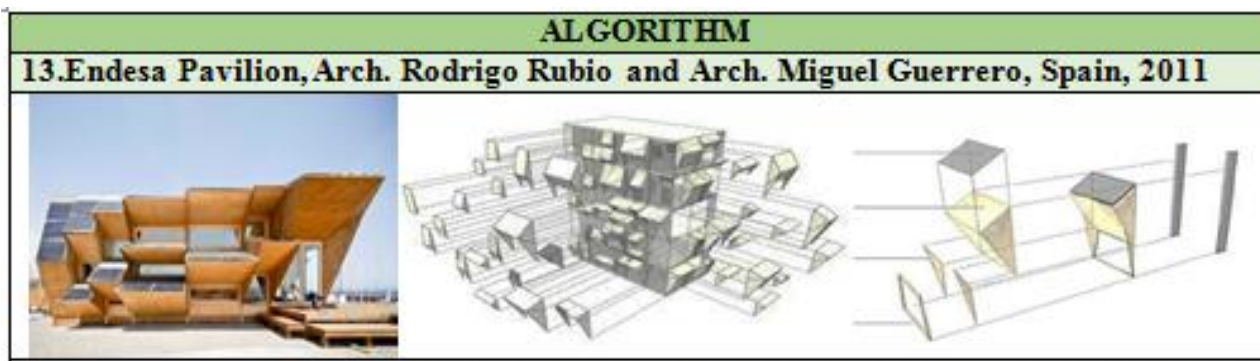

A facade composed by modular components, like solar brick, that react to photovoltaic gaining, solar protection, insulation, ventilation, lighting. The parametric rationality adapts façade geometries to the environmental requirements for each building's point. It calculated the building's optimal form based on sun's movement, solar slope and the structure's orientation [44]

14.The Museum of The Future, Killa Design, Dubai, United Arab Emirates, 2019

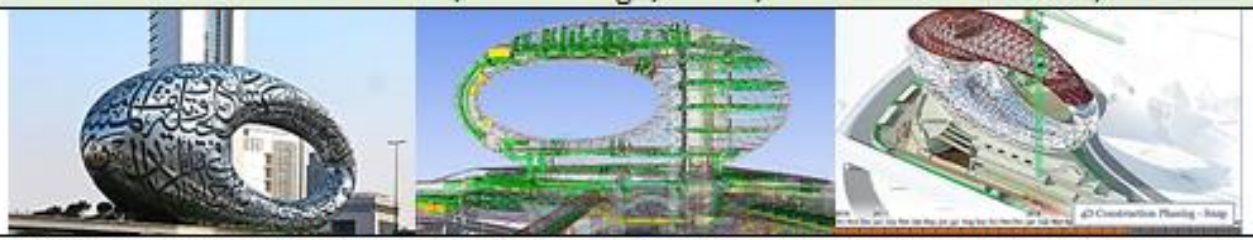

Inscriptions from Sheikh Mohammed's Arabic poetry repeated in pattems across its shiny exterior facade. Light enters the building through the perforated text, bathing visitors in the glowing Arabic script, with a massive hologram to fill the structure's oval-shaped void [45]

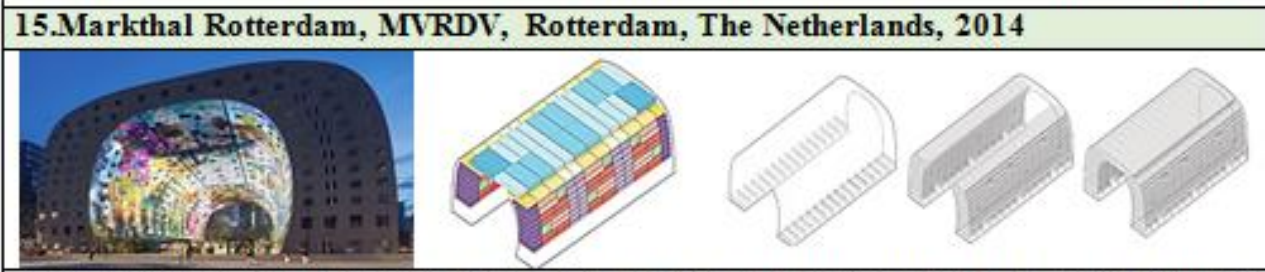

It was combined two housing buildings with an arched indoor market hall. An algorithmic parameterized software was utilized to generate different design solutions by changing parameter values [46]

16.Hotel Prestige Forest, Enric Ruiz Geli, Barcelona, Spain ,2009
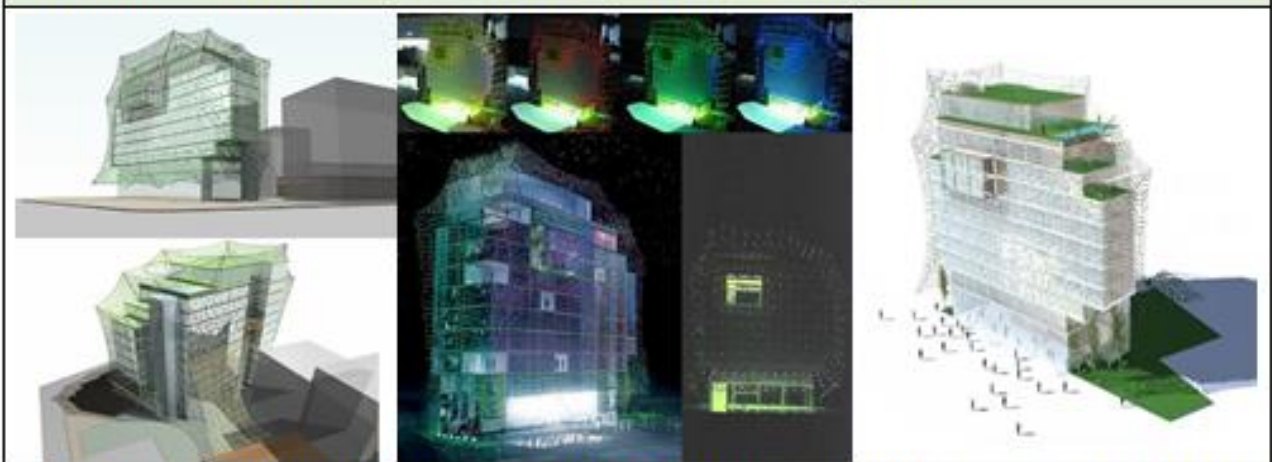

A stainless-steel mesh with artificial "leaves" that contain photovoltaic cells, with a battery, algorithmic CPU and a three-colour LED to absorb energy and convert into stored power [11] 
Table. 2. (Cont.)

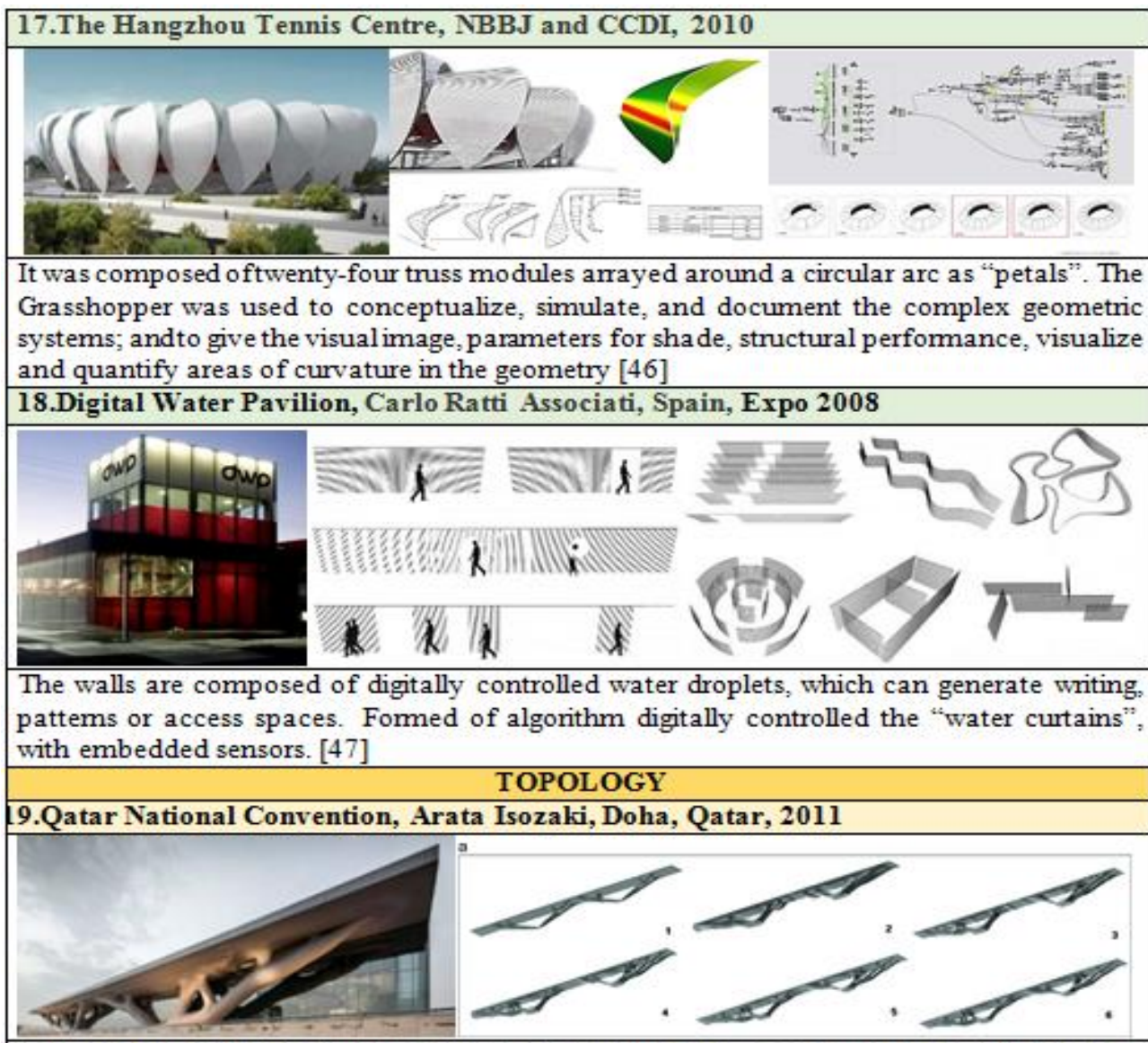

It was a huge organic structure like two intertwined trees gave the sense of the icon in Qatari culture. The tree structural form was created by Topology Optimization, that optimize the material densities [11]

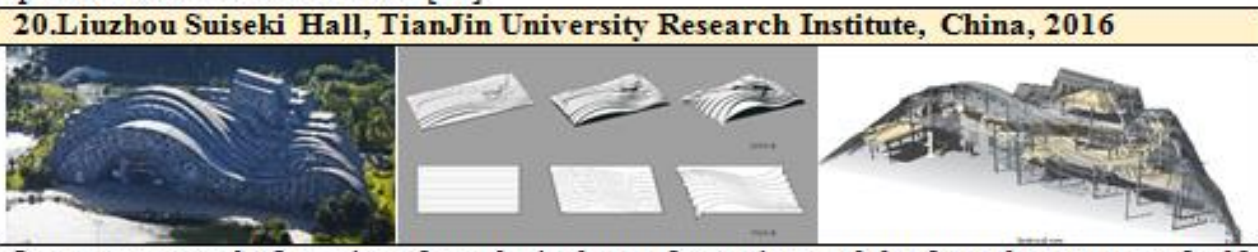

It was composed of a series of topological transformation and the fractal geometry of selfsimilar and self-a ffine randomvariation, one elevation is composed of water-like curve while the other one is composed of rock-like poly line, with a harmonious look [48]

21.The Island City Central Park, Toyo Ito, JAPAN, 2005

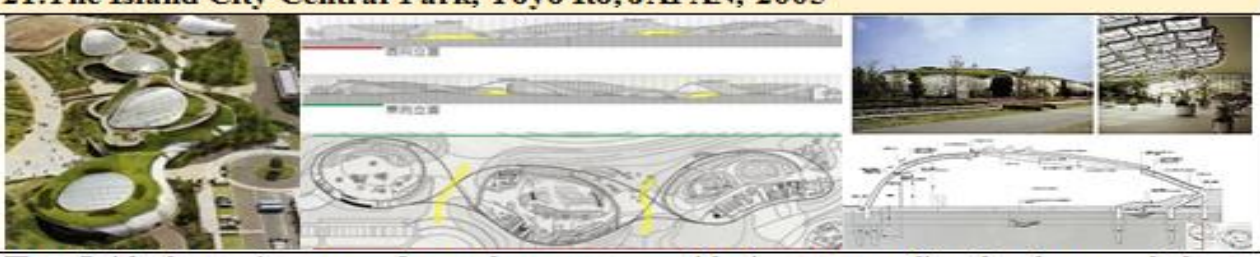

The fluid dynamic wave form that merges with its surrounding landscape, help to emphasize on the site cognition and preservation, to adjust the design parameters, and to seek out the best solutions that satisfies the mechanical conditions, and to enclose the maximum volume with minimum material [11] 
Table. 2. (Cont.)

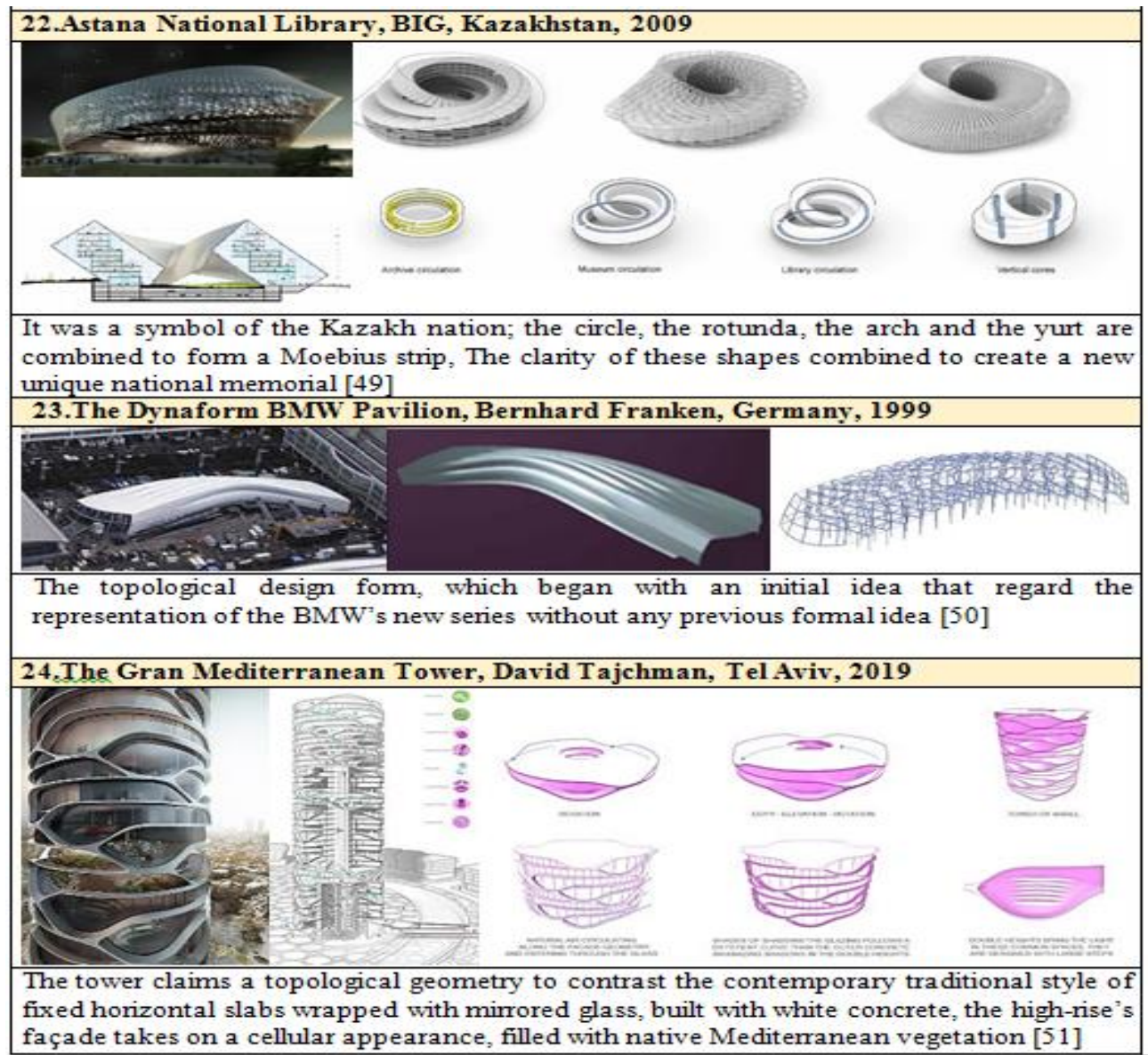

\section{Results and discussion}

The following parts represent the results of the questionnaire. They discuss the opinion and preference of the experts and the case studies.

\subsection{Case studies analysis}

Fig. 5 presents the results obtained from the preliminary analysis of the case studies. As shown, across all responses there is a significant difference between the four groups.

It was cleared that the Parametric type achieved the distinguished percentages with little differences between its case studies (50\%) to (35\%), followed by Geometry (45\%) to (35\%), then Topology (42\%) to (25\%). Although the Algorithm reached the highest percentages but with great differences between its case studies (53\%) to (23\%), which strengthened that the parametric type design tool could offer more features for buildings helping in reaching a better aesthetic performance. 


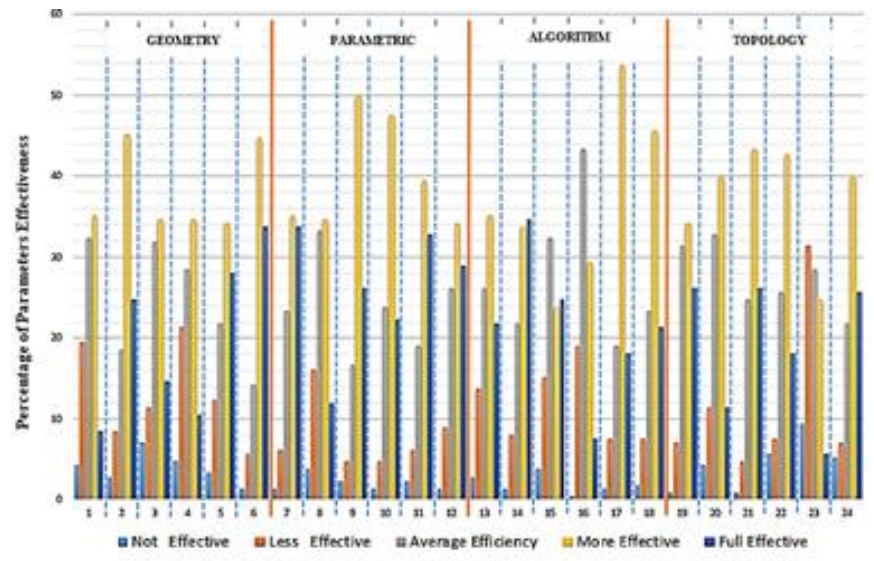

Fig. 5. The Comparative Analysis of The Case Studies [Author]

\subsection{The Correlation between mathematics and aesthetics' factorss}

From type of shape perspective, Fig. 6 displays the comparative analysis between four mathematics types as follows;

Parametric shape has a high percentage with "More Effective" in all aesthetic performance factors; "Form and Beauty" (45\%), "Vision and Perception" (43\%), Environmental (42\%) and "Implication Meaning" (33\%). Followed by Topology with the same sequence of factors (42\%, $36 \%, 35 \%, 34 \%$ ), then Geometry and Algorithm respectively.

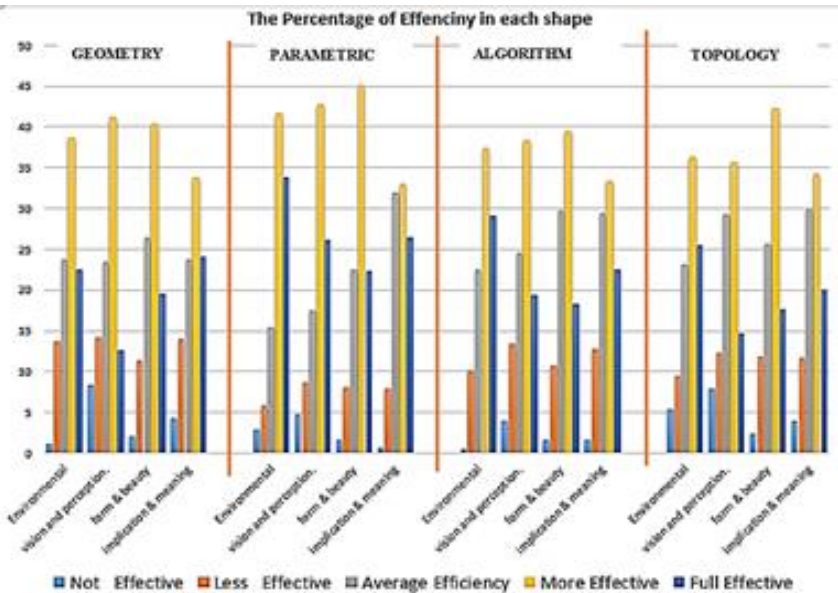

Fig. 6. The Comparative Analysis Between Four Mathematics Types [Author]

From category of aesthetic performance factors viewpoint, Fig. 7 illustrates the comparative analysis of the four aesthetic performance factors;

Environmental factor attained the highest percentage with "Full effective" in all shapes with 28\%, followed by Implication and Meaning with 23\%, Form and Beauty and Vision and Perception with $(19 \%, 18 \%)$ respectively. While Form and Beauty had the highest percentage in "More Effective" with (42\%), followed by Vision and Perception (40\%) and Environmental (38 \%) then Implication and Meaning (33\%).

On the other hand, Form and Beauty had the least percentage in "Not Effective" with (2\%) followed by Implication and Meaning (2.7\%) in all shapes. 


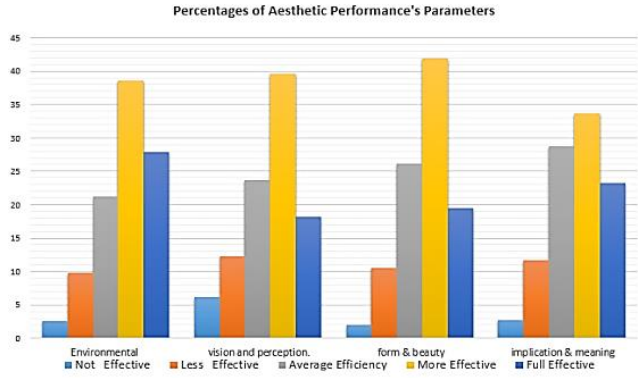

Fig. 7. The Comparative Analysis Of The Four Aesthetic Performance Factors [Author]

\section{Conclusion}

The present study determined the effect of utilizing mathematics fields as a design tool to achieve a good level of Aesthetic Performance. Results have shown that;

- Parametric design concept is the best one that could achieve most of the aesthetic performance factors. While the Topology design concept is the least one.

- Whereas the "Form and Beauty performance" is the most factor could be attained by most of design concepts, followed by the "Environmental performance". While the least one was the "Implication and Meaning performance". Thus, Fig. 8 clarified the concluded arrangement of the performance's degree of aesthetic performance factors in each mathematic type.

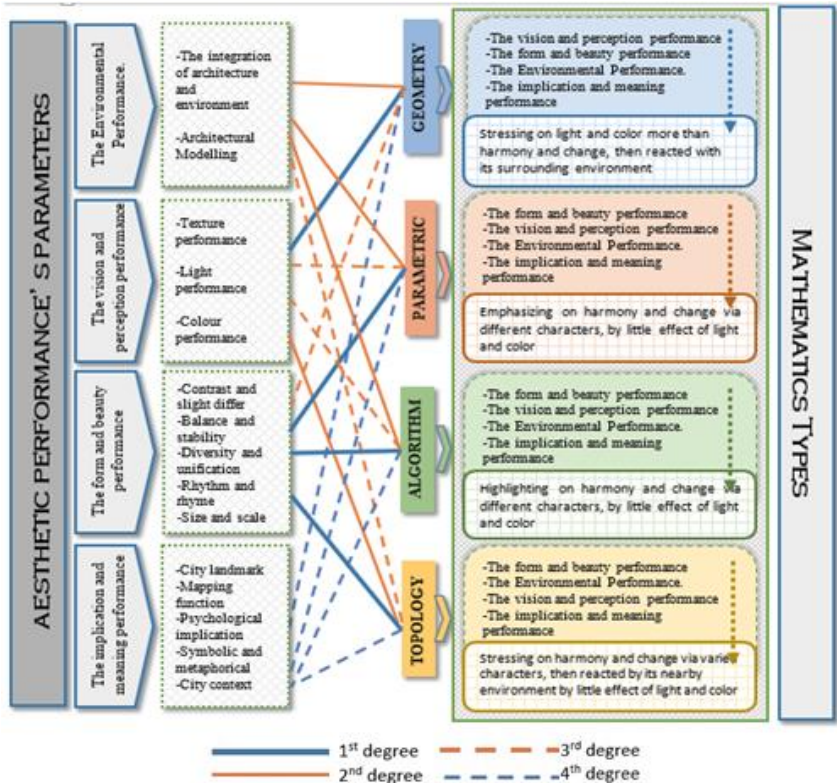

Fig. 8. Arrangement of The Performance's Priority in Each Form Type

\section{Recommendations and future work}

- Studying mathematics helps in developing creative abilities, skills and thinking and collaborating with visual thinking that could contribute in improving in innovative buildings design or redesign of the greatest buildings by considering its feature. 
- Architecture Students must be supplied with advanced architecture software that based on mathematics scripting which had a great role firstly in the creative design process, then in the preparing the working drawings.

- The focus of mathematical design teaching in the 21 st century needs moving to development-oriented design process that covers not only the aesthetical and functional dimensions but also the awareness and response of sensual and culture is indications.

- Further studies in this field of architecture design could be used to bridging the knowledge gaps easily by increasing the architect's role by offering them variable alternatives on ways of applying (design and presentation skills) of these contemporary approaches by investigating the ways of integrating mathematics' different branches, according to projects various requirements, for reaching building's optimum performance.

- Engineering faculties and education institutions should prepare forthcoming architecture designers with new curricula that integrate knowledge and understanding effectively the mathematics frameworks in their study in various architectural courses by a good knowledge of basic linear algebra and calculus that is considered great advantages in design.

- Must gain a prior understanding of both the cultural and social features which help in emphasizing on the architect's design concept and providing an opportunity to expand his point of view in applying mathematics.

\section{REFERENCES}

[1] Stavric, M. and Marina, O., "Parametric Modelling for Advanced Architecture", International Journal of Applied Mathematics and Informatics, Issue 1, Volume 5, P.9-16, 2011

[2] El Iraqi, A., "Form Generation in Architecture Using Tools Based on Evolutionary and Mathematical Functions", Master Thesis, Department of Architecture, Ain Shams University, 2008.

[3] SHI, X., "Performance-Based and Performance-Driven Architectural Design and Optimization", Higher Education Press and Springer-Verlag Berlin Heidelberg, 4(4): pp.512-518, 2010. DOI 10.1007/s11709-010-0090-6

[4] Albayrak, C., "Performative Architecture as a Guideline for Transformation of the Defence Line of Amsterdam", Master of science, Middle East Technical University Delft University of Technology, 2011

[5] Seyfzadeh, M. "The Mysterious Pyramid on Elephantine Island: Possible Origin of the Pyramid Code". Archaeological Discovery. 05. 187-223. 10.4236/ad.2017.54012., 2017

[6] Alexander J. Hahn, "Mathematical Excursions to the World's Great Buildings", Princeton University Press, 2012.

[7] https://www.archdaily.com/422470/ad-classics-the-guggenheim-museum-bilbao-frank-gehry (15/5/2019)

[8] http://www.mvsarchitects.com.au/doku.php?id=home:projects:victorian_college_of_the_arts (15/5/2019)

[9] https://www.arthitectural.com/zaha-hadid-architects-bogota-international-convention-centre/ (17/5/2019)

[10] Veizaj, D., Maliqari, A., "Expanding of The Limits of Forms in Architecture Through the Use of Parametric Methods and Algorithms", 1st International Conference On Architecture \& Urban Design Proceedings 19-21, pp.37:46, April 2012

[11] Burry, J. and Burry, M., "The New Mathematics of Architecture ", Thames and Hudson, 2010.

[12] Leopold, C., “Geometry Concepts in Architectural Design", 12th International Conference on Geometry and Graphics (ISGG), 2006.

[13] Choudhary, A., Dogne, N. and Maheshwari, S., "Mathematics and Architecture: Importance of Geometry", Ncaict: National Conference On Advances in Information and Communication Technology, December 2014 
[14]Gawell, E., "Non-Euclidean Geometry in the Modelling of Contemporary Architectural Forms", Journal of Polish Society for Geometry and Engineering, Graphics, Volume 24, pp. 35: 43, 2013.

[15] Alexander, I., Schröder, P., "Discrete Differential Geometry", Birkhäuser, p.5, 2008.

[16] Pottmann, H. and Wallner, J., "Freeform Architecture and Discrete Differential Geometry", International Conference on Discrete Geometry for Computer Imagery (DGCI), P.3-8, 2017.

[17] Crane, K. and Wardetzky, M., "A Glimpse into Discrete Differential Geometry", Notices of the American Mathematical Society Journal, Vol 64, No. 10, P.1153, 2017.

[18] Stotz, I., "Iterative Geometric Design for Architecture", Federal Institute of Technology in Lausanne, PhD Thesis, pp.20: 26, 2009

[19] https://infoscience.epfl.ch/record/142215/files/EPFL_TH4572.pdf (9/3/2018)

[20] Yehuda E., “Architecture's New Media: Principles, Theories, and Methods of Computer-Aided Design", The MIT Press, p.47, 2004.

[21] Kinayoglu, G., "A Reconsideration of the Concept of Architectural Space in The Virtual Realm", Middle East Technical University, Master of Architecture, p.42,43, 2007

[22] Rokicki, W., Gawell, E., "Voronoi Diagrams - Rod Structure Research Models In Architectural And Structural Optimization”, MAZOWSZE Studia Regionalne, Issue, 19, pp. 155:164, 2016

[23] Dino, I., "Creative Design Exploration by Parametric Generative Systems in Architecture", METU JFA 2012/1, pp. 207-224 DOI:10.4305/METU.JFA.2012.1.12

[24] http://www.italianways.com/riminis-malatesta-temple-emblem-of-the-renaissance/ (25/12/2018)

[25] Srinivasan, U., "Approaches to The Use of Geometry in Architecture: A Study of the Works of Andrea Palladio, Frank Lloyd Wright, and Frank Gehry", Master Thesis, Texas A\&M University, 2009.

[26] https://www.archdaily.com/89849/canton-tower-information-based-architecture (31/7/2018)

[27] Migliari, R., De Rosa, A., Rossi, M., and Ranon, R., "Descriptive Geometry and Digital Representation: Memory and Innovation", McGraw-Hill, 2012.

[28] https://www.arup.com/projects/yas-hotel (22/1/2019)

[29] https://www.dezeen.com/2009/12/07/the-tote-by-serie-architects/ (1/8/2018)

[30] Yousif, S., Clayton, M., and Yan, W., "Towards Integrating Aesthetic Variables in Architectural Design Optimization", 106th ACSA Annual Conference: The Ethical Imperative, March 2018.

[31] Miller, N., "The Hangzhou Tennis Centre: A Case Study in Integrated Parametric Design", ACADIA 2011: Parametricism.

[32] https://www.math.hmc.edu/ gu/curves_and_surfaces/surfaces/klein.html (4/8/2018).

[33] https://www.archdaily.com/777495/arnhem-central-transfer-terminal-unstudio (23/12/2018)

[34] Oxman, R., "Performance-Based Design: Current Practices and Research Issues", International Journal of Architectural Computing, 2008.

[35] DOI: 10.1260/147807708784640090

[36] Wang, X., "Research on Performance Evaluation of Architectural Aesthetics with the AHP Theory", Third International Conference on Intelligent System Design and Engineering Applications, p. 1185- 1190, 2013.

[37] https://www.dezeen.com/2013/10/03/museum-of-the-history-of-polish-jews-by-lahdelmamahlamaki-architects/ 22/4/2019

[38] https://www.e-architect.co.uk/africa/melaku-center-ethiopia (28/7/2018)

[39] https://www.archdaily.com/11602/shell-artechnic-architects (30/7/2018).

[40] http://www.hparc.com/work/the-grand-egyptian-museum/ (18/1/2019)

[41] Tsigkari, M., Davis, A. and Aish, F., Specialist Modelling Group, Foster + Partners, "A Sense of Purpose: Mathematics and Performance in Environmental Design", Architectural Design, Vol.81, No.4, 2011

[42] https://www.archdaily.com/778933/harbin-opera-house-mad-architects (22/1/2019) 
[43] https://www.archdaily.com/533388/south-australian-health-and-medical-research-institutewoods-bagot $(22 / 1 / 2019)$

[44] https://arcspace.com/feature/metropol-parasol/ (25/2/2019)

[45] http://www.ceramicarchitectures.com/obras/spanish-pavilion-expo-2005/ (26/1/2019).

[46] https://www.archdaily.com/274900/endesa-pavilion-iaac (17/2/2019).

[47] https://www.e-architect.co.uk/dubai/museum-of-the-future-in-dubai-building (20/2/2019)

[48] Feist, S., “A-BIM: Algorithmic-based Building Information Modelling”, Master Thesis, University of Lisbon, May 2016.

[49] https://carloratti.com/project/digital-water-pavilion/ (20/1/2019).

[50] https://www.archdaily.com/798941/liuzhou-suiseki-hall-tianjin-university-research-institute (25/2/2019)

[51] https://www.archdaily.com/33238/national-library-in-astana-kazakhstan-big (17/2/2019)

[52] Peschke, M., “Applying Generative Modelling Tools to Explore Architectural Forms.", The Journal of Polish Society for Geometry and Engineering Graphics Vol. 23, 2012.

[53] https://www.designboom.com/architecture/david-tajchman-gran-mediterraneo-mixed-usetower-tel-aviv-israel-04-04-2016/ (25/2/2019)

\section{الرياضيات كأداة تصميمة: نحو أداء جمالي فعّال للمبنى}

$$
\text { الملخص العربي }
$$

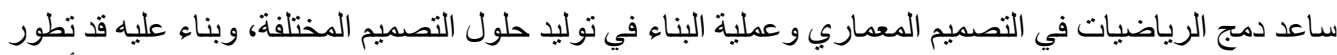

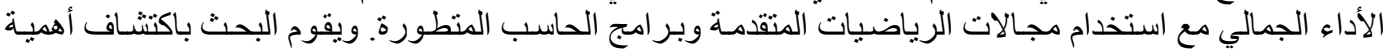

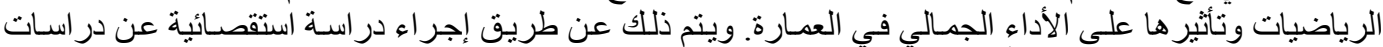

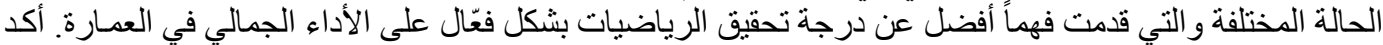

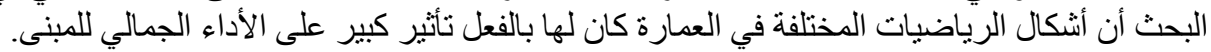

